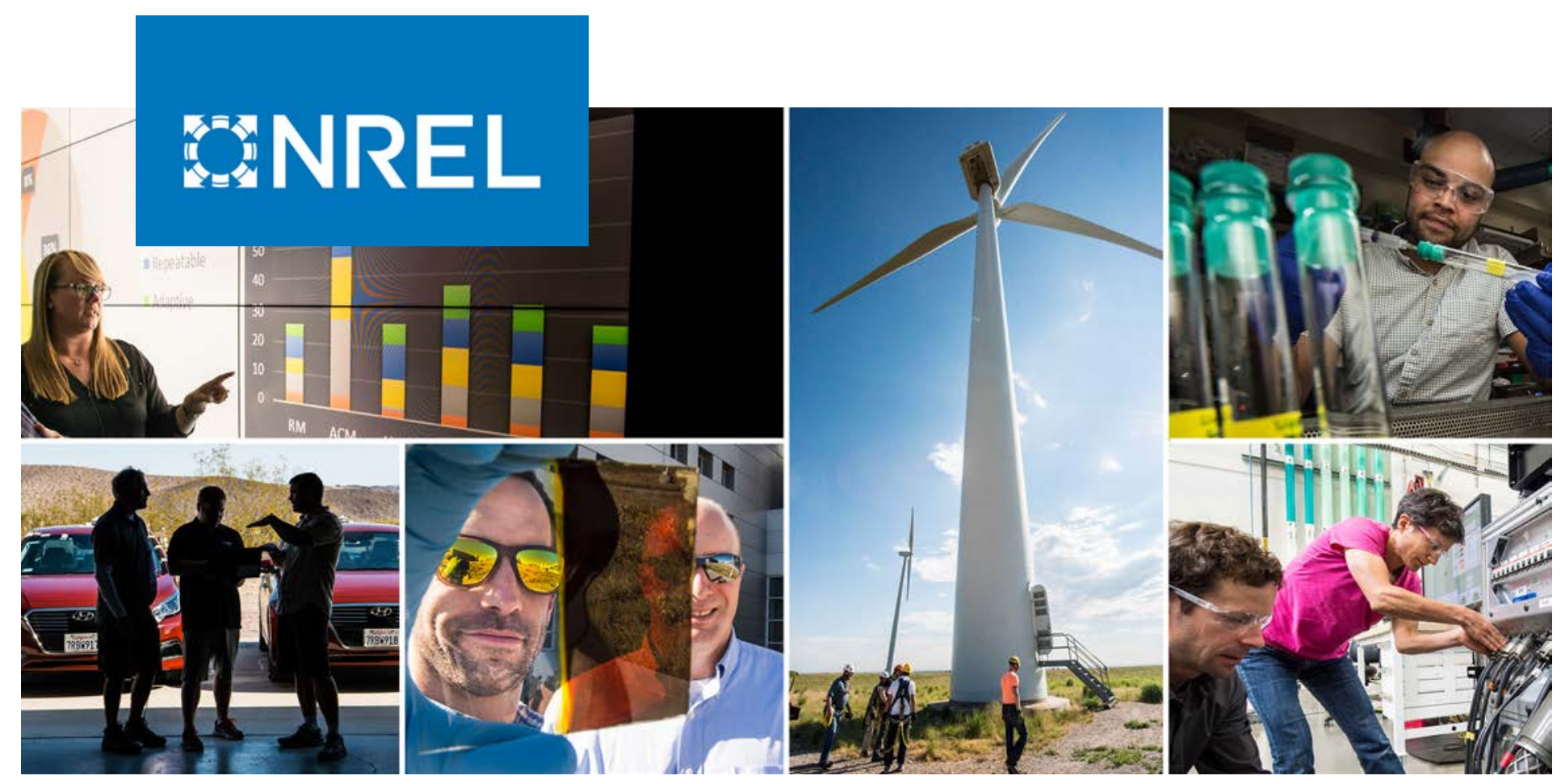

\title{
Multifidelity Uncertainty Quantification with Applications in Wind Turbine Aerodynamics
}

\section{Preprint}

Julian A. Quick, ${ }^{1}$ Peter E. Hamlington, ${ }^{1}$ Ryan N. King, ${ }^{2}$ and Michael A. Sprague ${ }^{2}$

1 University of Colorado, Boulder

2 National Renewable Energy Laboratory

Presented at the American Institute of Aeronautics and Astronautics SciTech Forum 2019

San Diego, California

January 7-11, 2019

NREL is a national laboratory of the U.S. Department of Energy

Office of Energy Efficiency \& Renewable Energy

Operated by the Alliance for Sustainable Energy, LLC

This report is available at no cost from the National Renewable Energy Laboratory (NREL) at www.nrel.gov/publications.
Conference Paper

NREL/CP-5000-72974

January 2019 


\title{
GNREL
}

\section{Multifidelity Uncertainty Quantification with Applications in Wind Turbine Aerodynamics}

\section{Preprint}

\author{
Julian A. Quick, ${ }^{1}$ Peter E. Hamlington, ${ }^{1}$ Ryan N. King, ${ }^{2}$ \\ and Michael A. Sprague ${ }^{2}$ \\ 1 University of Colorado, Boulder \\ 2 National Renewable Energy Laboratory
}

\section{Suggested Citation}

Quick, Julian A., Peter E. Hamlington, Ryan N. King, Michael A. Sprague. 2019.

Multifidelity Uncertainty Quantification with Applications in Wind Turbine Aerodynamics:

Preprint. Golden, CO: National Renewable Energy Laboratory. NREL/CP-5000-72974.

https://www.nrel.gov/docs/fy19osti/72974.pdf.

NREL is a national laboratory of the U.S. Department of Energy Office of Energy Efficiency \& Renewable Energy Operated by the Alliance for Sustainable Energy, LLC

This report is available at no cost from the National Renewable Energy Laboratory (NREL) at www.nrel.gov/publications.

Contract No. DE-AC36-08GO28308
Conference Paper

NREL/CP-5000-72974

January 2019

National Renewable Energy Laboratory 15013 Denver West Parkway Golden, CO 80401

303-275-3000 • www.nrel.gov 


\section{NOTICE}

This work was authored in part by the National Renewable Energy Laboratory, operated by Alliance for Sustainable Energy, LLC, for the U.S. Department of Energy (DOE) under Contract No. DE-AC36-08GO28308. Funding provided by the U.S. Department of Energy Office of Energy Efficiency and Renewable Energy Wind Energy Technologies Office. The views expressed herein do not necessarily represent the views of the DOE or the U.S. Government. The U.S. Government retains and the publisher, by accepting the article for publication, acknowledges that the U.S. Government retains a nonexclusive, paid-up, irrevocable, worldwide license to publish or reproduce the published form of this work, or allow others to do so, for U.S. Government purposes.

This report is available at no cost from the National Renewable Energy Laboratory (NREL) at www.nrel.gov/publications.

U.S. Department of Energy (DOE) reports produced after 1991 and a growing number of pre-1991 documents are available free via www.OSTI.gov.

Cover Photos by Dennis Schroeder: (clockwise, left to right) NREL 51934, NREL 45897, NREL 42160, NREL 45891, NREL 48097, NREL 46526.

NREL prints on paper that contains recycled content. 


\title{
Multifidelity Uncertainty Quantification with Applications in Wind Turbine Aerodynamics
}

\author{
Julian A. Quick* and Peter E. Hamlington ${ }^{\dagger}$ \\ Department of Mechanical Engineering, University of Colorado, Boulder, CO, 80309 \\ Ryan N. King \\ Computational Science Center, National Renewable Energy Laboratory, Golden, CO, 80401 \\ Michael A. Sprague ${ }^{\S}$ \\ National Wind Technology Center, National Renewable Energy Laboratory, Golden, CO, 80401
}

The propagation of input uncertainty through engineering models allows designers to better understand the range of possible outcomes resulting from design decisions. This could lead to greater trust between modelers and stakeholders in the wind energy industry. In this study, we apply multilevel-multifidelity Monte Carlo sampling to flow over an airfoil, assuming uncertainty in the inflow conditions, and characterize the associated computational savings compared to standard Monte Carlo approaches. The "truth" model is provided by an airfoil simulation with a very fine computational time step, and auxiliary lower-level models are provided by simulations with coarser time steps. Reynolds-averaged Navier Stokes and detached eddy simulations are used to obtain two different model fidelities. The primary quantity of interest for this analysis is the lift force, which is examined for a range of angles of attack. We launch an initial set of "trial" samples to determine the optimal allocation of model evaluations, and these trial evaluations are used to inform a larger sampling effort. Using the multilevel-multifidelity approach, we achieve roughly an order of magnitude variance reduction in expected lift as compared to the standard Monte Carlo approach with an equivalent computational cost.

\section{Nomenclature}

$\alpha=$ blending parameter for detached eddy simulation model

$\alpha_{l} \quad=$ multilevel-multifidelity control variate associated with level $l$

$\alpha_{l}^{*} \quad=$ optimal multilevel-multifidelity control variate associated with level $l$

$C_{a}^{b} \quad=$ evaluation time associated with fidelity $b$ at discretization $a$

$\tilde{C}=$ computational budget

Cov $=$ covariance operator

$\delta_{i j} \quad=$ Kronecker delta

$d V=$ differential volume

$d S \quad=$ differential surface

$\epsilon \quad=$ sampling error tolerance

$\mathbb{E} \quad=$ expected value operator

$f \quad=$ quantity of interest

$f_{a}^{b}=$ quantity of interest associated with model fidelity $b$ at discretization level $a$

$f_{i} \quad=$ body force

$F \quad=\quad$ wall blending function

$g \quad=$ gravity vector

$h \quad=$ enthalpy

*Research Assistant, 427 UCB, Boulder, CO, 80309, AIAA Student Member

$\dagger$ Assistant Professor, 427 UCB, Boulder, CO, 80309, AIAA Member

¥ Senior Scientist, 15013 Denver West Parkway, Golden, CO, 80401, AIAA Member

${ }^{\S}$ Senior Scientist, 15013 Denver West Parkway, Golden, CO, 80401, AIAA Member 


$\begin{array}{ll}K & =\text { turbulence kinetic energy } \\ l & =\text { discretization level } \\ L & =\text { finest discretization level } \\ N & =\text { number of model evaluations } \\ N_{l} & =\text { number of model evaluations associated with discretization level } l \\ N_{k}^{\text {ip }} & =\text { interpolation point for node } k \\ n_{j} & =\text { standard normal vector } \\ v & =\text { uniform, nonnegative, and constant viscosity } \\ \mu_{i j}^{t} & =\text { turbulent viscosity } \\ \phi & =\text { generic interpolation variable } \\ \phi & =\text { generic uncertain input } \\ P & =\text { pressure perturbation from the thermodynamic pressure } \\ Q^{\mathrm{MLMF}} & =\text { multilevel-multifidelity Monte Carlo estimator } \\ \rho & =\text { air density } \\ \rho_{0} & =\text { ambient air density } \\ \rho_{l} & =\text { Pearson correlation coefficient between low- and high-fidelity models using discretization } l \\ r_{l} & =\text { difference between the number of low- and high-fidelity samples associated with discretization } l \\ S_{i j} & =\text { rate of strain tensor } \\ t & =\text { time field } \\ \tau_{i j}^{\text {sgs }} & =\text { subgrid turbulent stress } \\ u_{i} & =\text { velocity vector } \\ \text { Var } & =\text { variance operator } \\ x_{i} & =\text { spatial vector } \\ y & =\text { distance from nearest wall } \\ Y_{a} b & =\text { multilevel correction function associated with model fidelity } b \text { at discretization level } a\end{array}$

\section{Introduction}

Wind energy systems are incredibly complex, spanning a wide range of spatio-temporal scales and involving interactions between atmospheric boundary layer physics, blade aerodynamics, turbine structural dynamics, and wake flow physics. In the face of this complexity, supercomputing resources are required to numerically predict the flow through a wind power plant using a high-fidelity model. Directly quantifying the effects of uncertainty in material properties, flow measurements, and operating conditions in such high-fidelity models introduces an additional, currently prohibitive, computational cost.

Consequently, it is not currently feasible to evaluate a high-fidelity wind power plant model enough times to characterize the range of possibilities arising from the myriad of uncertainties associated with the physical system. Several techniques have been proposed for overcoming this difficulty, including stratified sampling techniques [1] and polynomial approximations of the response surface [2-4]. Despite these advances, however, efficiently propagating uncertainty through complex systems described by partial differential equations with high-dimensional uncertainty remains a challenging problem.

As a solution, computationally cheaper and less accurate (i.e., lower-fidelity) models may be fused with a computationally demanding but more accurate (i.e., high-fidelity) model to produce an estimate of a statistic of a quantity of interest [5]. One example of the multifidelity uncertainty quantification approach is to use a surrogate model to approximate the discrepancy between low- and high-fidelity models [6-8]. Multifidelity Monte Carlo sampling using the control variate approach has recently emerged as a new method of variance reduction, which is not plagued by the curse of dimensionality [9, 10]. Geraci et al. [11, 12] introduced the multilevel-multifidelity Monte Carlo approach, which allows for simultaneous fusion of different discretization choices and models with different fidelities.

In this study, we explore the computational savings associated with multilevel-multifidelity approaches by sampling across different turbulence models and different temporal resolutions for flow over an airfoil, assuming uncertainty in the incoming flow speed and angle of attack. We use unsteady Reynolds-averaged Navier-Stokes (RANS) and detached eddy simulation (DES) turbulence closures as our low- and high-fidelity models respectively, and identify sets of different temporal resolutions for each turbulence model to be used as the levels in the multilevel-multifidelity uncertainty quantification setting. The models are implemented in Nalu-Wind [13], a wind-focused fork of Sandia National Laboratory's Nalu [14] code base. Here, we apply the multilevel-multifidelity uncertainty quantification 
technique to two-dimensional flow across the DU 91-W2-250 airfoil. We examine the RANS shear-stress transport (SST) and DES turbulence models, using the time-step size as a model resolution parameter. We compare the computational efficiency of the multilevel-multifidelity estimator to the conventional Monte Carlo approach with the expected airfoil lift force as our quantity of interest. To explore this methodology, we tested the multifidelity-multilevel approach using unusually wide probability densities. Ultimately, this study is intended as a precursor to the full multilevel-multifidelity quantification of uncertainty in a high-fidelity model of a wind energy power plant.

It should be noted that there have been similar studies examining stochastic turbulent flow in a multifidelity setting [15-18]. Pisaroni et al. [8] presented a continuum multilevel approach to quantifying the range of possible outcomes of stochastic two- and three-dimensional flows using RANS models with different spatial resolutions. Geraci et al. [12] used multilevel-multifidelity Monte Carlo to quantify a range of possibilities of flow through a nozzle. Witteveen et al. [2] used stochastic collocation to quantify the response of a transonic airfoil to a range of possible inflow conditions. Padrón et al. [3] used polynomial chaos expansion to construct a surrogate discrepancy between low- and high-fidelity wind turbine models to estimate the range of possible impacts of a gust with uncertain inflow characteristics. Recently, Maniaci et al. [19] used the multilevel-multifidelity approach to fuse the FAST.FARM low-fidelity wind power plant model with several discretizations of the Nalu high-fidelity wind power plant model, simulating a wind turbine with periodic inflow/outflow boundary conditions to mimic the wake effect of upstream turbines.

The remainder of this paper is structured as follows. Section $\Pi$ describes the framework used to analyze propagation of uncertainty using the multilevel-multifidelity approach, turbulence modeling, temporal convergence analysis, and the numerical experiments performed. Section $[\mathrm{IV}$ presents the variance reduction achieved with the multifidelity approach and compares the computational costs and expected errors to the conventional multifidelity estimators using high-fidelity and reduced-order models. These results and future work are then discussed in Section $\mathbf{D}$.

\section{Methodology and Application}

In this study, the multilevel-multifidelity sampling approach is applied to two-dimensional flow over an airfoil, assuming uncertainty in the inflow magnitude and angle of attack. An analysis of the time-step resolution is carried out to assess the temporal resolution needed to resolve important features in the evolution of the flow field, and this resolution level is identified as the truth discretization. More coarsely resolved time discretizations are identified as displaying similar general trends, but with lower computational costs. A temporal convergence analysis is performed by plotting lift coefficient versus angle of attack for varying time-step sizes. Monte Carlo sampling is performed on the different models and the resulting sample set is used to inform full sampling efforts for the multilevel-multifidelity estimator.

\section{A. Variance Reduction Technique}

The multilevel-multifidelity Monte Carlo method is used to relate correlated models, reducing variance in the estimate of the expectation of the more expensive, high-fidelity model. The standard Monte Carlo estimator for an expectation from a model is given by

$$
\mathbb{E}[f(\boldsymbol{\phi})] \approx \frac{1}{N} \sum_{i=1}^{N} f\left(\boldsymbol{\phi}_{i}\right),
$$

where $f$ represents a quantity of interest from the high-fidelity model and $N$ represents the number of samples used to make the estimate. The parameters, $\boldsymbol{\phi}_{i}$, are sampled randomly from a prescribed distribution to form the Monte Carlo estimator. For a situation where the function $f$ is itself not exact and can be constructed using different discretization levels, we can define the new random variable, $Y_{l}$, as

$$
Y_{l}(\boldsymbol{\phi})= \begin{cases}f_{1}(\boldsymbol{\phi}) & \text { if } l=1, \\ f_{l}(\boldsymbol{\phi})-f_{l-1}(\boldsymbol{\phi}) & \text { if } l>1,\end{cases}
$$

where $l=1, \ldots L$ represents the discretization level, with $f_{1}$ representing the coarsest discretization and $f_{L}$ the finest discretization. The corresponding expectation for the finest discretization is then obtained from Eq.1 1 using a telescoping sum as

$$
\mathbb{E}\left[f_{L}(\boldsymbol{\phi})\right] \approx \sum_{l=1}^{L} \frac{1}{N_{l}} \sum_{i=1}^{N_{l}} Y_{l}\left(\boldsymbol{\phi}_{i}\right),
$$


where $N_{1} \geq \cdots \geq N_{L}$ represents the number of samples at each discretization level. Note that as $\left(N_{1}, \ldots, N_{L}\right) \rightarrow$ $(\infty, \ldots, \infty)$, the estimator approaches the true expectation.

The control variate approach may be used to further decrease the computational burden of the Monte Carlo estimator. In multilevel-multifidelity Monte Carlo simulation, the control variate is applied to the differences between coarse and fine resolutions for low- and high-fidelity models; namely

$$
\mathbb{E}\left[f_{L}^{\mathrm{HF}}(\boldsymbol{\phi})\right] \approx Q^{\mathrm{MLMF}}=\sum_{l=1}^{L}\left\{\frac{1}{N_{l}} \sum_{k=1}^{N_{l}} Y_{l}^{\mathrm{HF}}\left(\boldsymbol{\phi}_{k}\right)+\alpha_{l}\left[\frac{1}{\left(1+r_{l}\right) N_{l}} \sum_{i=1}^{\left(1+r_{l}\right) N_{l}} Y_{l}^{\mathrm{LF}}\left(\boldsymbol{\phi}_{i}\right)-\sum_{k=1}^{N_{l}} \frac{1}{N_{l}} Y_{l}^{\mathrm{LF}}\left(\boldsymbol{\phi}_{k}\right)\right]\right\},
$$

where $Q^{\mathrm{MLMF}}$ is the multilevel-multifidelity Monte Carlo estimator, and $r_{l} \geq 0$ is the difference between the number of low- and high-fidelity model evaluations at discretization level $l$. Typically, $N_{L}$ will be quite small, since the high-fidelity model is expensive to evaluate, and $N_{L}$ is typically substantially less than $N_{1}$.

The value of the parameter $\alpha_{l}$ in the above expression for $Q^{\mathrm{MLMF}}$ is determined by minimizing its variance, which is given by

$$
\operatorname{Var}\left(Q^{\mathrm{MLMF}}\right)=\sum_{l=1}^{L}\left\{\frac{1}{N_{l}} \operatorname{Var}\left(Y_{l}^{\mathrm{HF}}[\boldsymbol{\phi}]\right)+\frac{r_{l}}{\left(1+r_{l}\right) N_{l}}\left[\alpha_{l}^{2} \operatorname{Var}\left(Y_{l}^{\mathrm{LF}}[\boldsymbol{\phi}]\right)-2 \alpha_{l} \operatorname{Cov}\left(Y_{l}^{\mathrm{HF}}\left[\boldsymbol{\phi}_{k}\right], Y_{l}^{\mathrm{LF}}[\boldsymbol{\phi}]\right)\right]\right\}
$$

The minimization is performed by setting $d \operatorname{Var}\left(Q^{\mathrm{MLMF}}\right) / d \alpha_{l}$ equal to zero, where

$$
\frac{d \operatorname{Var}\left(Q^{\mathrm{MLMF}}\right)}{d \alpha_{l}}=\frac{r_{l}}{\left(1+r_{l}\right) N_{l}}\left[2 \alpha \operatorname{Var}\left(Y_{l}^{\mathrm{LF}}[\boldsymbol{\phi}]\right)-2 \operatorname{Cov}\left(Y_{l}^{\mathrm{HF}}[\boldsymbol{\phi}], Y_{l}^{\mathrm{LF}}[\boldsymbol{\phi}]\right)\right]
$$

The resulting value of $\alpha_{l}$ that gives the minimum variance is then obtained as

$$
\alpha_{l}^{*}=\frac{\operatorname{Cov}\left(Y_{l}^{\mathrm{HF}}[\phi], Y_{l}^{\mathrm{LF}}[\phi]\right)}{\operatorname{Var}\left(Y_{l}^{\mathrm{LF}}[\phi]\right)}
$$

Because $d^{2} \operatorname{Var}\left(Q_{\mathrm{CV}}\right) / d \alpha^{2}=2 \frac{r_{l}}{\left(1+r_{l}\right) N_{l}} \operatorname{Var}\left(Y_{l}^{\mathrm{LF}}\left[\phi_{i}\right]\right)$ is non-negative, we can say that $\alpha_{l}^{*}$ indeed minimizes $\operatorname{Var}\left(Q_{\mathrm{CV}}\right)$.

Previous works [19-21] derive the optimal allocation of high-fidelity samples as

$$
N_{l}=\frac{2}{\epsilon}\left\{\sum_{k=1}^{L}\left[\frac{\operatorname{Var}\left(Y_{k}^{\mathrm{HF}}\right) C_{k}^{\mathrm{HF}}}{1-\rho_{k}^{2}}\right]^{1 / 2} \Lambda_{k}\left(r_{k}\right)\right\} \sqrt{\left(1-\rho_{l}^{2}\right) \frac{\operatorname{Var}\left(Y_{l}^{\mathrm{HF}}\right)}{C_{l}^{\mathrm{HF}}}}
$$

where $\epsilon$ is an error tolerance, $\Lambda_{l}=1-\frac{r_{l}}{1+r_{l}} \rho_{l}^{2}, \rho_{l}$ is the correlation between $f_{l}^{\mathrm{HF}}$ and $f_{l}^{\mathrm{LF}}$ over the prescribed probability distribution of $\phi$, and $r_{l}$ is calculated as

$$
r_{l}=\sqrt{\frac{\rho_{l}^{2}}{\left(1-\rho_{l}^{2}\right)} \frac{C_{l}^{\mathrm{HF}}}{C_{l}^{\mathrm{LF}}}}-1
$$

where $C_{l}^{\mathrm{HF}}$ and $C_{l}^{\mathrm{LF}}$ are the costs of the high- and low-fidelity models associated with level $l$, respectively. It is noted that this does not take variability in computational cost into account.

The optimal number of level-specific high-fidelity model evaluations may also be expressed as a function of the number of finest-level high-fidelity model evaluations. With this formulation, the optimal number of model evaluations is independent of $\epsilon$ and is given by

$$
\frac{N_{l}^{\mathrm{HF}}}{N_{i}^{\mathrm{HF}}}=\sqrt{\frac{\left(1-\rho_{l}^{2}\right) \operatorname{Var}\left(Y_{l}^{\mathrm{HF}}\right) C_{i}^{\mathrm{HF}}}{\left(1-\rho_{i}^{2}\right) \operatorname{Var}\left(Y_{i}^{\mathrm{HF}}\right) C_{l}^{\mathrm{HF}}}}
$$

\section{B. Sample Allocation Strategy}

The total computational cost $\tilde{C}$ may be computed as

$$
\tilde{C}=N_{L} \sum_{j=0}^{L} \frac{N_{j}}{N_{L}}\left[C_{j}^{\mathrm{HF}}+\left(1+r_{j}\right) C_{j}^{\mathrm{LF}}\right]
$$


where $\frac{N_{j}}{N_{L}}$ is defined (according to Equation 10 as

$$
\frac{N_{j}}{N_{L}}=\sqrt{\frac{\left(1-\rho_{j}^{2}\right) \operatorname{Var}\left(Y_{j}^{\mathrm{HF}}\right) C_{L}^{\mathrm{HF}}}{\left(1-\rho_{L}^{2}\right) \operatorname{Var}\left(Y_{L}^{\mathrm{HF}}\right) C_{j}^{\mathrm{HF}}}} .
$$

Given a computational budget, $\tilde{C}$, we can compute the optimal number of fine-discretization high-fidelity samples,

$$
N_{L}=\frac{\tilde{C}}{\sum_{j=1}^{n} \frac{N_{j}}{N_{L}}\left[C_{j}^{\mathrm{HF}}+\left(1+r_{j}\right) C_{j}^{\mathrm{LF}}\right]},
$$

and the remaining number of high-fidelity samples can be calculated as $N_{j}=N_{L} \frac{N_{j}}{N_{L}}$. All DES model evaluations were associated with SST model evaluations that used the same random number generator seed. With the exception of the coarsest discretization, all model samples were associated with samples performed on the next-coarsest level, using the same random number generator seed. We ensured that samples were performed using the same seed using a dataframe, joining the data based on the inflow speed and angle of attack and enforcing that the low-fidelity sets of samples included the same samples used by the high-fidelity samples considered by the multilevel-multifidelity estimator. We used the ceiling operator whenever floating point numbers were converted to integers, rounding up.

In most settings, the correlation and variance statistics must be estimated. If there are not enough samples, the variance tends to be underestimated and the correlation tends to be overestimated. So, these statistics must be approximated in order to calculate the optimal allocation of model samples. In practice, it is unlikely that the optimal distribution of model evaluations would actually be used, as this calculation changes as more samples are evaluated. In this study, we mimic the real process by first reporting the results of a small trial set of samples then optimally fusing model evaluations for a large computational budget, considering the multifidelity-multilevel combination of the DES and RANS models, using time discretizations of $\mathrm{CFL}=10,000, \mathrm{CFL}=1,000$, and $\mathrm{CFL}=100$.

We first launched a set of trial samples across each model to explore the model correlation and variance spaces. We prescribed five initial evaluations of the most expensive model and evaluated the remaining models in parallel with similar computational time. The number of trial simulations used are reported in Table 1 . All of these trial samples are also used in the full analysis. For larger studies, the computational budget could start at a prescribed value and gradually be increased to a prescribed final value, calculating a new value of $N_{l}$ and $r_{l}$ during each iteration and evaluating the newly prescribed samples.

We collected enough auxiliary model evaluations to optimally apply the multilevel-multifidelity estimator. We report time convergence plots, comparing the standard deviation and mean estimate from each estimator to the standard Monte Carlo approach. Additionally, we plot the estimated error and standard deviation as functions of time. We estimated the true expectation using the Chaospy package's stochastic collocation tool [22] using Tikhonov regularization. We used all samples available from the trial and full sampling stages to inform the $\alpha_{l}$ value used in the analysis, effectively assuming a priori knowledge about the variance and covariance statistics.

\section{Turbulence Modeling}

In the present study, we chose two turbulence models for the different model fidelities: the SST unsteady RANS model (low fidelity) and a DES model (high fidelity), which uses the RANS SST model in regions close to walls and a large eddy simulation model in free-stream locations. Both models are used to close the low-Mach fluid flow equations, which are given as [13]

$$
\begin{gathered}
\int \frac{\partial \bar{\rho}}{\partial t} d V+\int \bar{\rho} \tilde{u}_{j} n_{j} d S=0, \\
\int \frac{\partial \bar{\rho} \tilde{u_{i}}}{\partial t} d V+\int \bar{\rho} \tilde{u}_{i} \tilde{u}_{j} n_{j} d S=\int\left[\left(2 \mu \tilde{S}_{i j}^{*}-\bar{P} \delta_{i j}\right) n_{j}-\tau_{i j}^{\mathrm{sgs}} n_{j}\right] d S+\int\left[\left(\bar{\rho}-\rho_{0}\right) g_{i}+f_{i}\right] d V, \\
\int \frac{\partial \bar{\rho} \tilde{h}}{\partial t} d V+\int \bar{\rho} \tilde{h} \tilde{u}_{j} n_{j} d S=-\int\left[\bar{q}_{j} n_{j}+\tau_{h, j}^{\mathrm{sgs}} n_{j}\right] d S+\int\left[-\frac{\partial \bar{q}_{i}^{r}}{\partial x_{i}}+\left(\frac{\partial \bar{P}}{\partial t}+\tilde{u}_{j} \frac{\partial \bar{P}}{\partial x_{j}}\right)+\overline{\left(2 \mu \tilde{S}_{i j}^{*}\right) \frac{\partial u_{i}}{\partial x_{j}}}+S_{\theta}\right] d V,
\end{gathered}
$$

where $u_{i}$ is the velocity vector, $\rho$ is the density, $\rho_{0}$ is the ambient density, $x_{i}$ is the spatial vector, $t$ is time, $h$ is the enthalpy, $q$ is the heat flux by conduction, $\bar{q}$ is the diffusive heat flux, $P$ represents pressure perturbations away from the 
thermodynamic pressure $\left(P_{\text {total }}=P_{\text {th }}+P\right), n_{i}$ is the normal vector, $\rho_{\infty}$ is the ambient density, $f_{i}$ is a body force vector, and $S_{\theta}$ is the energy source term. The thermodynamic pressure, $P_{\text {th }}$, is given by the ideal gas equation of state as

$$
P_{\mathrm{th}}=\rho \frac{R}{W} T,
$$

where $R$ is the universal gas constant, $W$ is the mean molecular weight of air, and $T$ is the average temperature. The deviatoric mean strain rate tensor, $\tilde{S}_{i j}^{*}$, is given as

$$
\tilde{S}_{i j}^{*}=\frac{1}{2}\left(\frac{\partial \tilde{u}_{i}}{\partial x_{j}}+\frac{\partial \tilde{u}_{j}}{\partial x_{i}}\right)-\frac{\delta_{i j}}{3} \frac{\partial \tilde{u}_{k}}{\partial x_{k}} .
$$

Note that, in the above equations, $\bar{\phi}$ represents an ensemble average of a generic variable, $\phi$, and $\tilde{\phi}$ is the Favre-average defined as

$$
\tilde{\phi} \equiv \frac{\overline{\rho \phi}}{\bar{\rho}} .
$$

Closure of the above equations requires models for the subgrid scale (SGS) terms appearing in the momentum and enthalpy equations, namely

$$
\begin{gathered}
\tau_{i j}^{\mathrm{sgs}}=\bar{\rho}\left(\widetilde{u_{i} u_{j}}-\tilde{u}_{i} \tilde{u}_{j}\right), \\
\tau_{h, j}^{\mathrm{sgs}}=\bar{\rho}\left(\widetilde{h u_{j}}-\tilde{h} \tilde{u}_{j}\right) .
\end{gathered}
$$

In the following, we describe the RANS and DES models used to close the momentum SGS term. For details about the enthalpy SGS term, we refer to the Nalu-Wind theory manual [13].

\section{RANS Model}

The RANS model applies an ensemble average to the governing equations and solves the resulting equations using a finite-volume formulation. The subgrid Reynolds stresses are modeled as

$$
\tau_{i j}^{\mathrm{sgs}} \approx \mu_{i j}^{t}\left(\frac{\partial \tilde{u_{i}}}{\partial x_{j}}+\frac{\partial \tilde{u}_{j}}{\partial x_{i}}-\frac{2}{3} \frac{\partial \tilde{u}_{k}}{\partial x_{k}}\right)-\frac{2}{3} \rho k \delta_{i j}
$$

where $k$ is the turbulence kinetic energy and $\mu_{i j}^{t}$ is the modeled turbulent viscosity.

A wall distance marker function is computed in preprocessing. The model uses the $k-\omega$ model close to walls and the $k-\epsilon$ model away from walls. The transport equations for these quantities are

$$
\begin{gathered}
\int \frac{\partial \bar{\rho} k}{\partial t} d V+\int \bar{\rho} k \tilde{u}_{j} n_{j} d S=\int\left(\mu+\hat{\sigma}_{k} \mu_{t}\right) \frac{\partial k}{\partial x_{j}} n_{j} d S+\int\left(P_{k}^{\omega}-\beta^{*} \bar{\rho} k \omega\right) d V \\
\int \frac{\partial \bar{\rho} \omega}{\partial t}+\int \bar{\rho} \omega \tilde{u}_{j} n_{j} d S=\int\left(\mu+\hat{\sigma}_{\omega} \mu_{t}\right) \frac{\partial \omega}{\partial x_{j}} n_{j} d S+\int\left[2(1-F) \frac{\bar{\rho} \sigma_{\omega_{2}}}{\omega} \frac{\partial k}{\partial x_{j}} \frac{\partial \omega}{\partial x_{j}}+\left(\frac{\hat{\gamma}}{v_{t}} P_{k}^{\omega}-\hat{\beta} \bar{\rho} \omega^{2}\right)\right] d V,
\end{gathered}
$$

where $\beta^{*}=0.09$ and the production $P_{k}^{\omega}$ is given by

$$
P_{k}^{\omega}:=\min \left(\mu_{i j}^{t} \frac{\partial \tilde{u}_{i}}{\partial x_{j}}\left(\frac{\partial \tilde{u}_{i}}{\partial x_{j}}+\frac{\partial u_{j}}{\partial x_{i}}\right), 10 \times \beta^{*} \bar{\rho} k \omega\right) .
$$

The coefficients $\hat{\sigma}_{k}, \hat{\sigma_{\omega}}, \hat{\gamma}$, and $\hat{\beta}$ are blended using the wall function, $F$. For a variable, $\hat{\phi}$, the blending is accomplished as

$$
\hat{\phi}=F \phi_{1}+(1-F) \phi_{2},
$$

where $\sigma_{k 1}=0.85, \sigma_{k 2}=1.0, \sigma_{\omega 1}=0.5, \sigma_{\omega 2}=0.856, \gamma_{1}=5 / 9, \gamma_{2}=0.44, \beta_{1}=0.075$, and $\beta_{2}=0.0828$. The blending function, $F$ is calculated as

$$
F=\tanh \left\{\min \left[\max \left(\frac{\sqrt{k}}{\beta^{*} \omega y}, \frac{500 \mu}{\bar{\rho} y^{2} \omega}\right), \frac{4 \bar{\rho} \sigma_{\omega 2} k}{\mathrm{CD}_{k \omega} y^{2}}\right]^{4}\right\},
$$


where $y$ is the distance from the nearest wall. The $\mathrm{CD}_{k \omega}$ parameter is calculated as

$$
\mathrm{CD}_{k \omega}=\max \left(2 \bar{\rho} \sigma_{\omega 2} \frac{1}{\omega} \frac{\partial k}{\partial x_{j}} \frac{\partial \omega}{\partial x_{j}}, 10^{-10}\right)
$$

and the turbulent viscosity is modeled as

$$
\mu_{i j}^{t}=\frac{a_{1} \bar{\rho} k}{\max \left(a_{1} \omega, \tilde{S}_{i j} F_{2}\right)},
$$

where the second blending function $F_{2}$, is given by

$$
F_{2}=\tanh \left[\max \left(\frac{2 \sqrt{k}}{\beta^{*} \omega y}, \frac{500 \mu}{\bar{\rho} \omega y^{2}}\right)^{2}\right] .
$$

\section{DES Model}

The DES model is mathematically similar to the SST model. The kinetic energy transport equation is modified to include a new dissipation scale. The modified equation is

$$
\int \frac{\partial \bar{\rho} k}{\partial t} d V+\int \bar{\rho} k \tilde{u}_{j} n_{j} d S=\int\left(\mu+\hat{\sigma}_{k} \mu_{t}\right) \frac{\partial k}{\partial x_{j}} n_{j} d S+\int\left(P_{k}^{\omega}-D_{k}\right) d V
$$

where $D_{k}=\rho k^{3 / 2} / l_{D}, l_{D}=\min \left(l_{S S T}, \hat{c}_{D E S} l_{D E S}\right)$, where $l_{S S T}=k^{1 / 2} / \beta^{*} \omega, \hat{c}_{D E S}$ is a blend between $c_{D E S_{1}}=0.78$ and $c_{D E S_{2}}=0.61$, and $l_{D E S}$ is the maximum edge length scale in contact with the node.

\section{Numerical Approach}

The equations described in the previous section were solved using Nalu-Wind [13]. This code supports control volume finite element method (CVFEM) and edge-based vertex-centered (EBVC) discretization approaches. The Nalu-Wind manual [13] recommends using the CVFEM to solve the continuity equation and the EBVC method for all other transport equations, which is the setting we used. The CVFEM approach constructs a dual mesh within the input grid. The current code base supports discretization of unstructured meshes to second-order accurate stencils using the dual mesh. It allows for higher-order dual-mesh constructions. The EBVC discretization approach uses the dual mesh to precompute dual mesh nodal volumes and edge-based area vectors to provide a stencil for second-order-accurate differentiation schemes.

In both cases, time is discretized using a second-order-accurate three-point backward-Euler stencil. A target Courant--Friedrichs-Lewy (CFL) number is specified to control the time-step size. The CFL number is the local velocity multiplied by the timestep size and divided by the characteristic mesh size. It is computed across the domain and the global CFL number is the maximum CFL number across the domain. The advection, diffusion, and stress tensor terms are discretized and Green's theorem is used to balance conservation laws in a large system of linear equations. The values are interpolated to the control volume surfaces to estimate flux terms. The advection term is stabilized by balancing a higher-order upwind derivative with an unstabilized central Galerkin differentiation.

The pressure is stabilized by augmenting the continuity equation with residuals from the momentum equation. The stabilized continuity equation is

$$
\int \frac{\partial \bar{\rho}}{\partial t} d V+\int\left(\bar{\rho} \hat{u}_{j}+\tau G_{j} \bar{P}\right) n_{j} d S=\int \tau \frac{\partial \bar{P}}{\partial x_{j}} n_{j} d S
$$

where $G_{j} \bar{P}$ is an $L_{2}$ nodal projection of the pressure gradient and $\hat{U}$ is the provisional velocity found using the momentum equation.

We used central differencing (as apposed to upwind differencing) in all our simulations. A nonlinear stabilization term is introduced, with a form similar to a discontinuity capturing operator (an artificial viscosity is computed to maintain stability, which is different than the turbulent viscosity) [13]. 


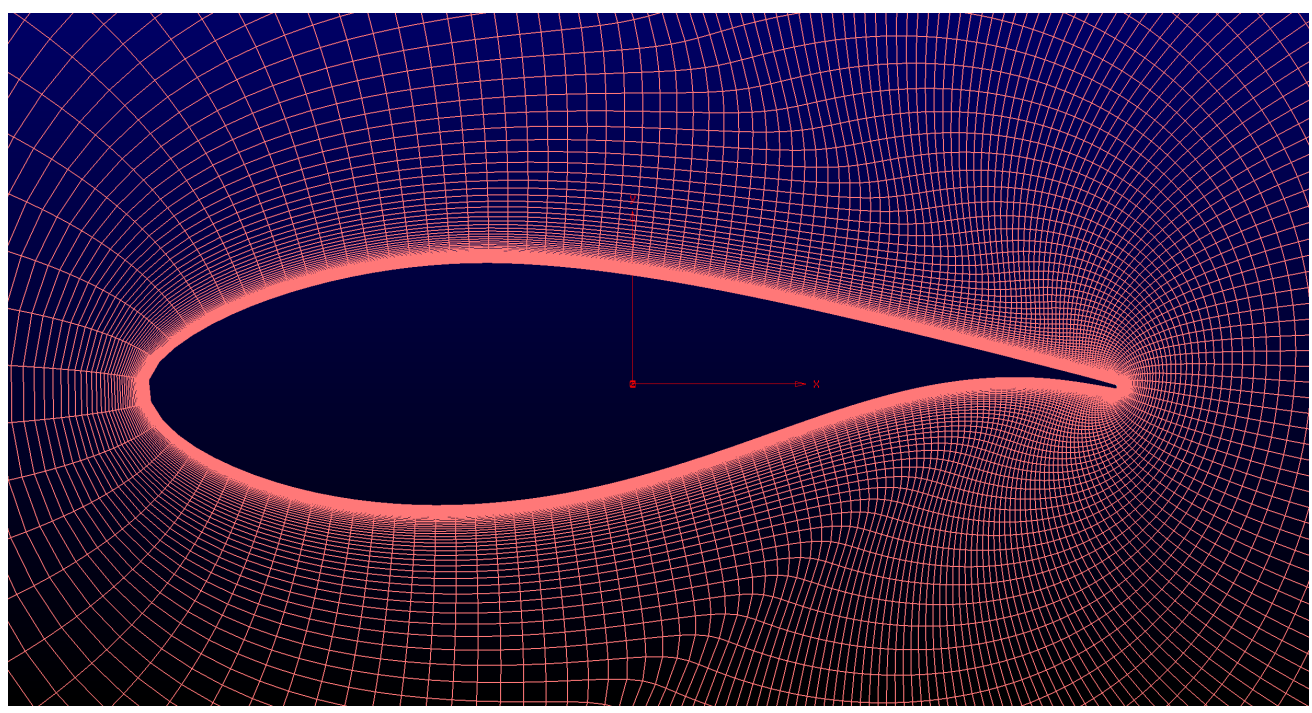

Fig. 1 Close-up of DU 91-W2-250 airfoil shape and the associated computational mesh.

\section{E. Test Case: DU 91-W2-250 Airfoil}

We tested the computational gains associated with the multifidelity-multilevel Monte Carlo strategy using twodimensional flow over an airfoil by comparing the computational efficiency of the approach to standard Monte Carlo. Specifically, we examined the DU 91-W2-250 airfoil, assuming uncertainty in the inflow speed and angle of attack. We chose this airfoil because it is representative of the midsection of a wind turbine blade. We decided to cast the turbulence model as simulation fidelity and the CFL number as the level parameter. The problem was solved using the Nalu-Wind framework [13, 14], as described in the previous section. We chose the average lift force (averaged between 6 and 10 seconds) as the quantity of interest. We prescribed inflow density of $1.177 \mathrm{~kg} / \mathrm{m}^{3}$ and viscosity of $1.177 \times 10^{-5} \mathrm{~kg} / \mathrm{ms}$.

The airfoil shape and grid are shown in Figure 1 . The mesh was generated with the PointWise extrusion function. The dimensionless wall distance, $y^{+}$, was set to a value of 0.3 and the other settings were left as default. The mesh has 100 points along each half of the airfoil and has a diameter of about 40 chord lengths. The mesh has a total of 30,502 degrees of freedom. This mesh was used in all simulations discussed here.

We assumed uncertainty in the inflow velocity and angle of attack to explore the multilevel-multifidelity concept. We decided to choose wide probability distributions as a test for this methodology. In future work, we plan to examine input distributions around an operating point. The angle of attack ranged uniformly from $0^{\circ}$ to $8^{\circ}$ and the inflow magnitude was modeled as a Weibull probability distribution with a scale factor of 30 and shape factor of 2.5; Figure 2 shows the distribution of wind speeds used in this study.

We first simulated a set of trial runs, only using five samples from the fine-discretization models. We sampled the remaining hierarchy of model discretizations using a similar amount of total computational effort. These trial runs were used to inform our full sampling efforts. Each set of samples was parallelized across several computational nodes. The samples were performed in serial on each node using the DAKOTA framework [24]. Increasing the inflow magnitude increases the amount of fine-scale motion, which corresponds to smaller time step sizes and greater computational burden. For each discretization and fidelity, we noted the minimum number of completed samples across all parallelized jobs and only collected that many samples from each completed sampling job, discarding the remaining samples. Without this step, our approach would naturally favor parameters associated with lower computational costs, and our samples would not all be identically distributed.

\section{F. Convergence Analyses}

A convergence analyses was performed to assess the required time resolution necessary to resolve important features of the flow field. In this study, we focused on angles of attack between $0^{\circ}$ and $8^{\circ}$. Time histories associated with $8^{\circ}$ angle of attack and $R e=6 \times 10^{6}$ are shown in Figures 3 and 4 for the SST and DES models, respectively. A summary of sweeps across CFL and angle of attack is shown for $R e=6 \times 10^{6}$ in Figure 5. Based on these results, we decided it was reasonable to cast $\mathrm{CFL}=100$ as well-resolved. As a result, we treated the DES model with CFL $=100$ as the 


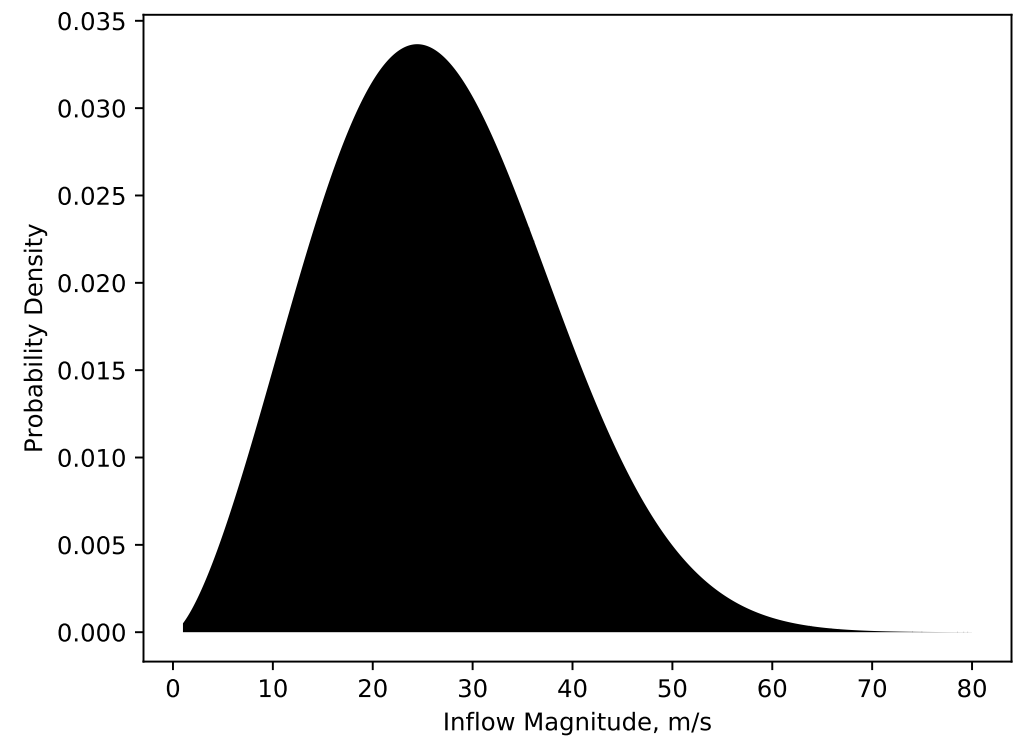

Fig. 2 Probability density of the inflow wind speed magnitudes considered in this study. This is not necessarily representative of actual uncertainty in practice, but is a useful distribution for testing the multilevel-multifidelity Monte Carlo approach.

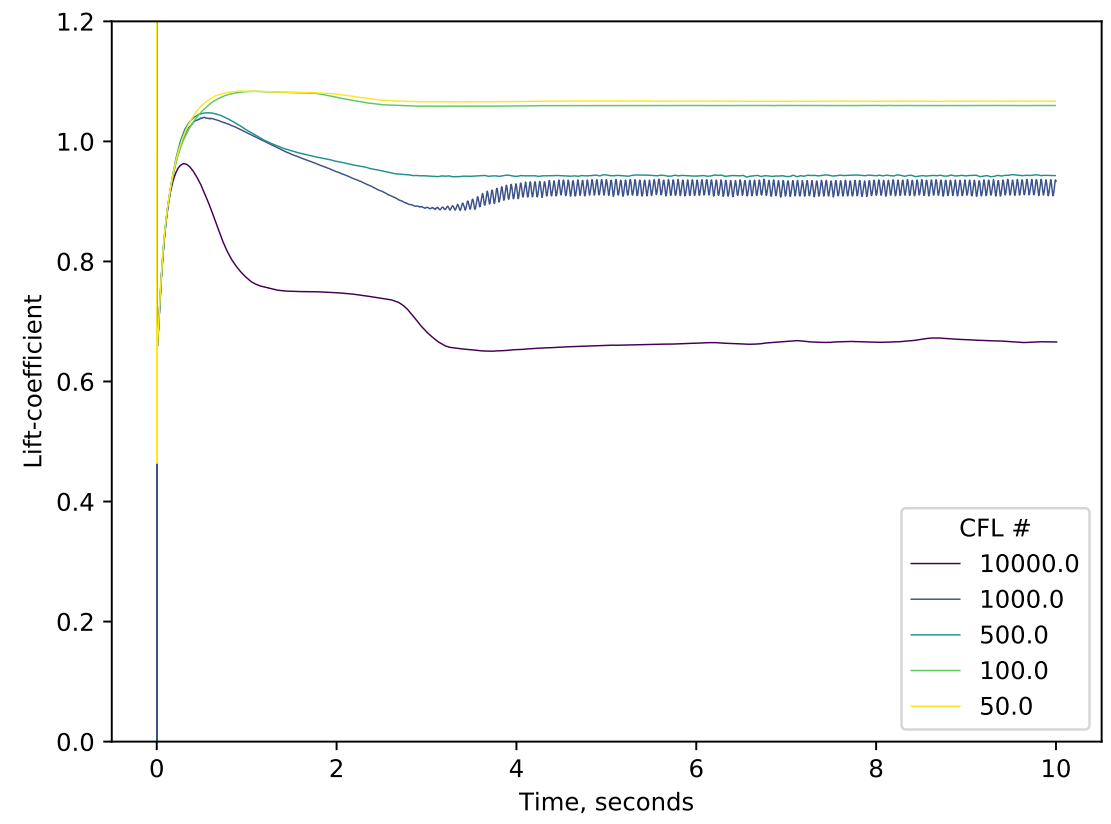

Fig. 3 Lift coefficient time history associated with the RANS SST turbulence model at $8^{\circ}$ angle of attack with $\operatorname{Re}=6 \times 10^{6}$.

"truth" case. Flow fields for the DES and SST models are shown for CFL $=100$ (Figure 6), CFL = 1, 000 (Figure 7), and $\mathrm{CFL}=10,000$ (Figure 8 ). 


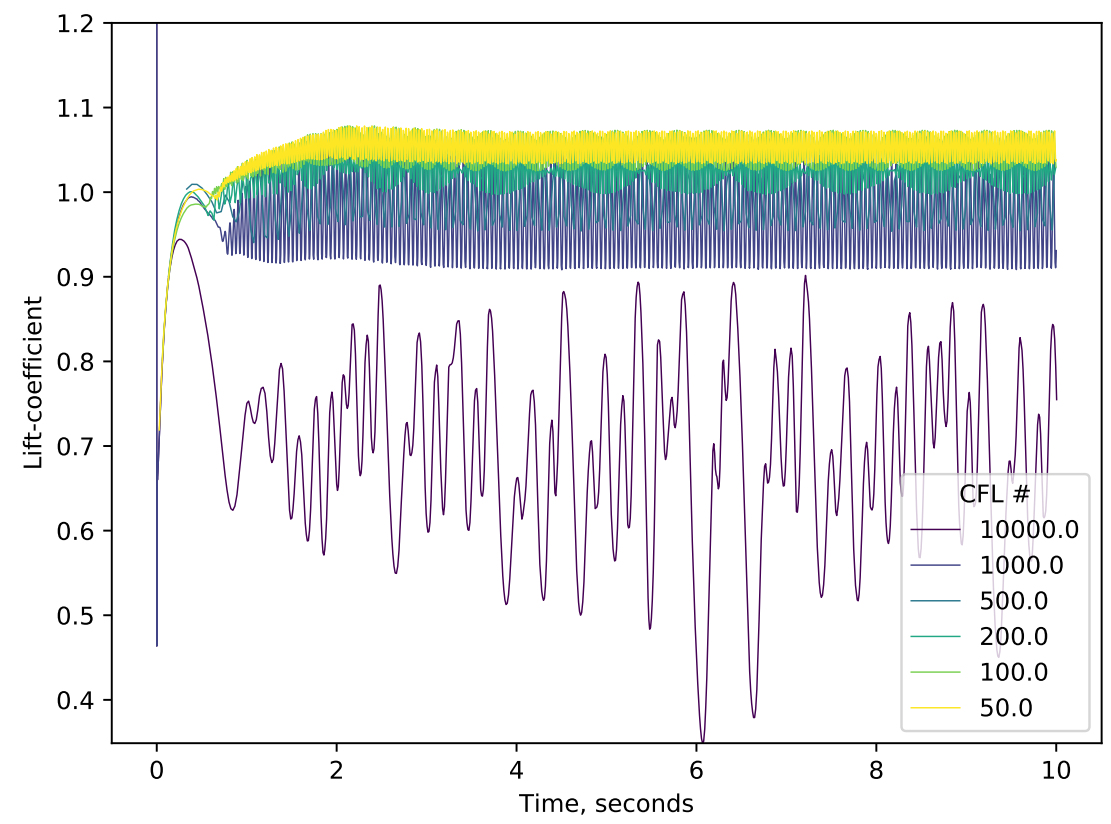

Fig. 4 Lift coefficient time history associated with the DES turbulence model at $8^{\circ}$ angle of attack with $\operatorname{Re}=6 \times 10^{6}$.

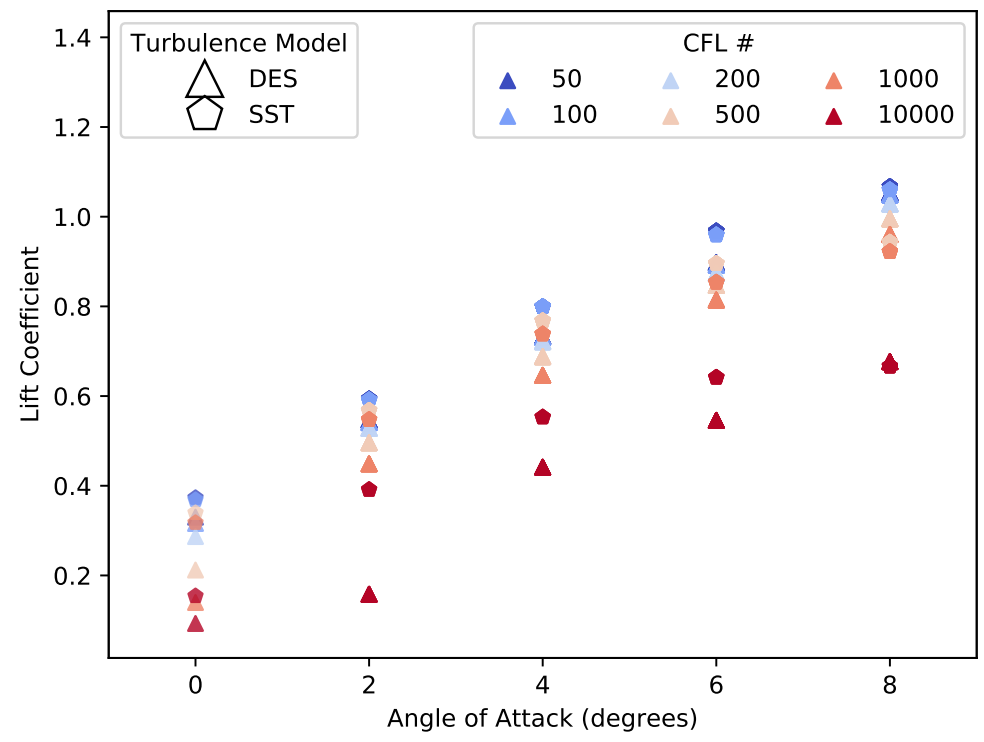

Fig. 5 Lift coefficient versus angle of attack for the RANS SST and DES models, exploring a range of CFL numbers. The simulations were performed at $R e=6 \times 10^{6}$. 


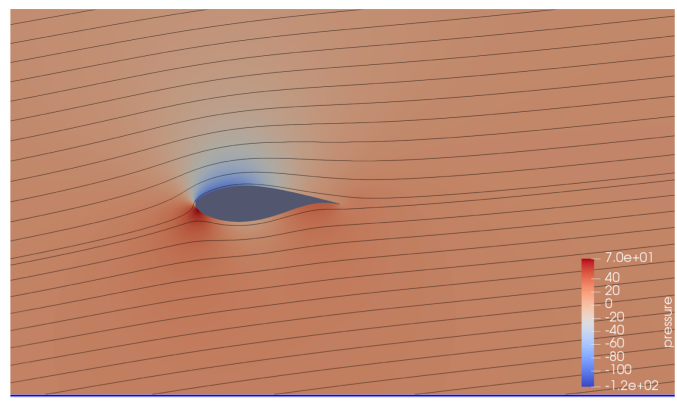

(a) SST Pressure Field

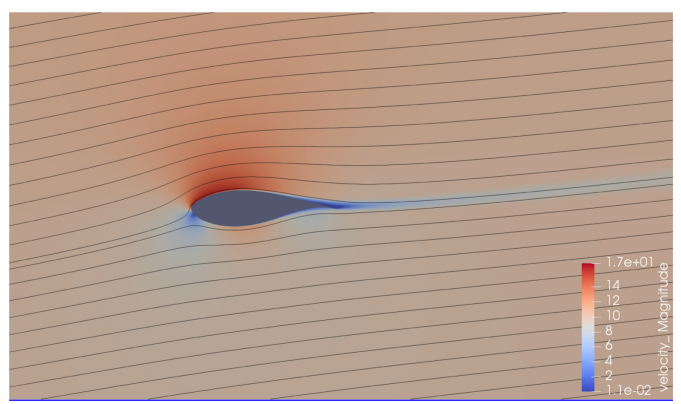

(c) SST Velocity Field

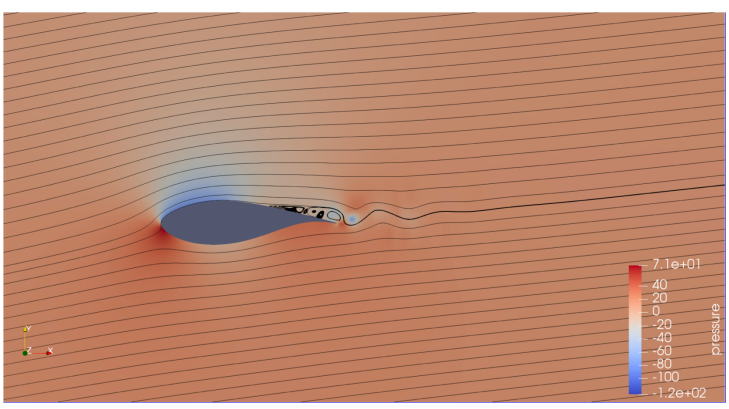

(b) DES Pressure Field

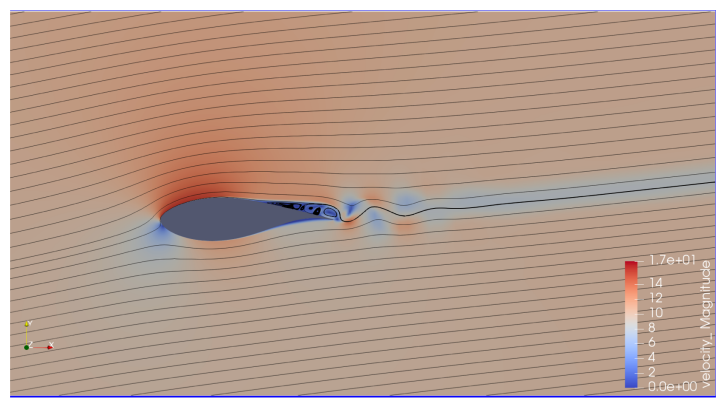

(d) DES Velocity Field

Fig. 6 Flow fields for CFL number of 100 and Reynolds number equal to $10^{6}$. The SST (left) and DES (right) model outputs are qualitatively compared using pressure (top) and velocity (bottom) fields.

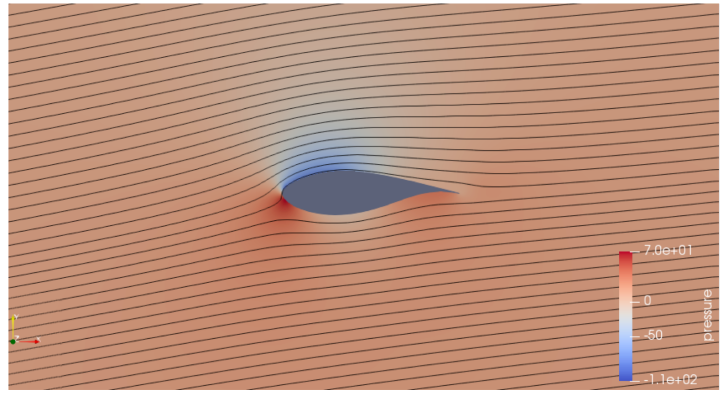

(a) SST Pressure Field

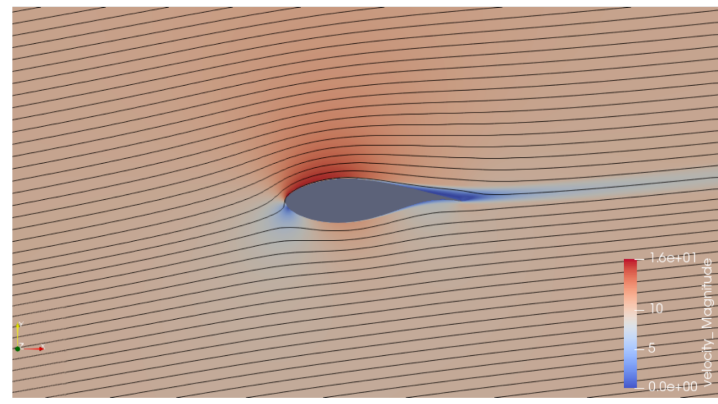

(c) SST Velocity Field

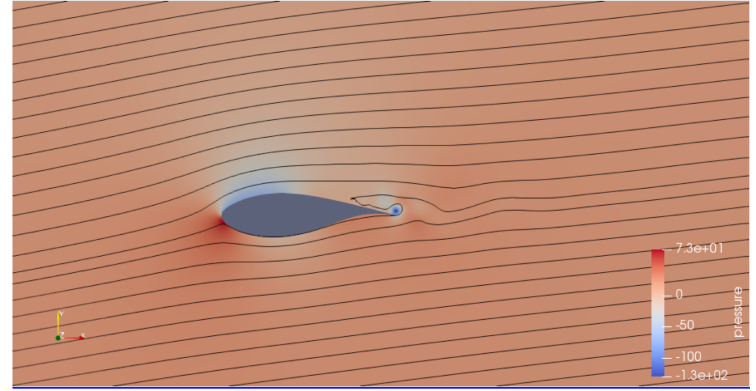

(b) DES Pressure Field

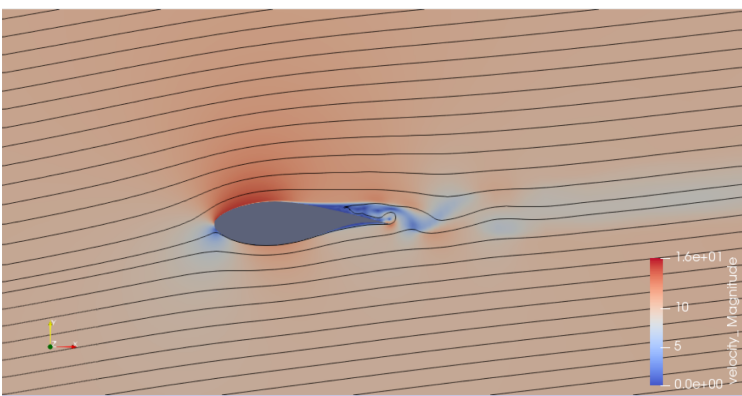

(d) DES Velocity Field

Fig. 7 Flow fields for CFL number of 1,000 and Reynolds number equal to $10^{6}$. The SST (left) and DES (right) model outputs are qualitatively compared using pressure (top) and velocity (bottom) fields. 


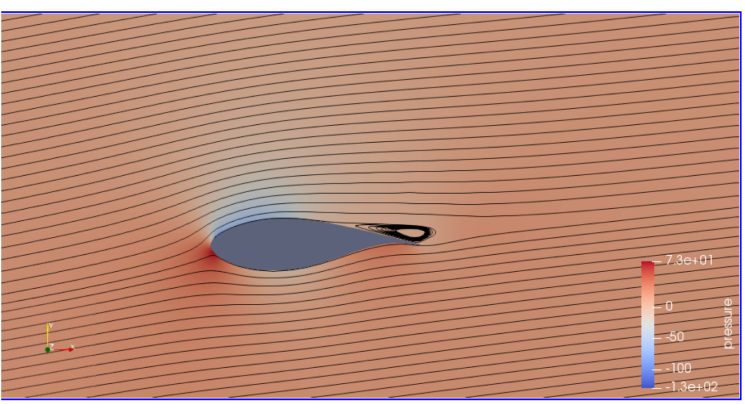

(a) SST Pressure Field

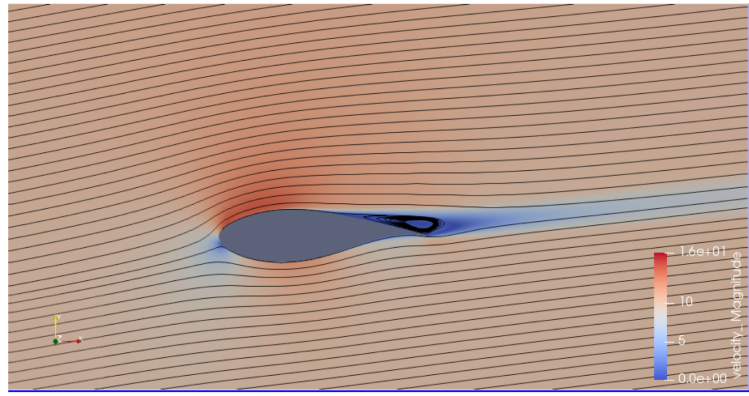

(c) SST Velocity Field

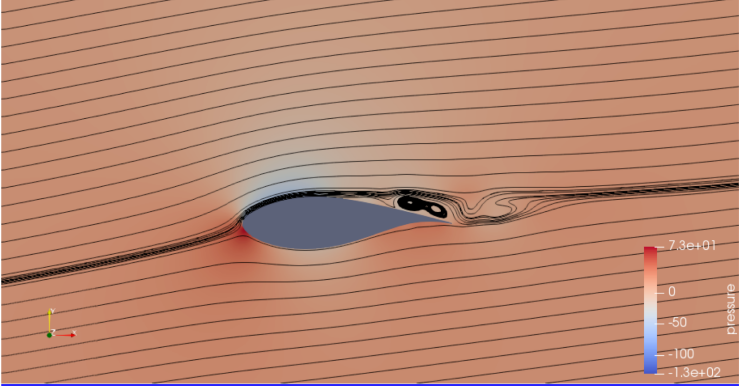

(b) DES Pressure Field

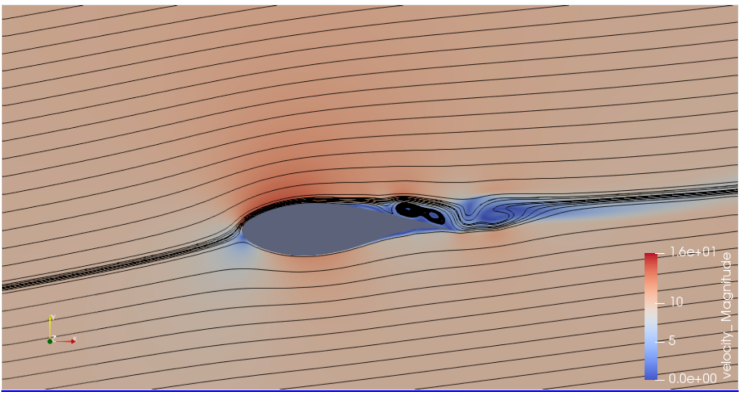

(d) DES Velocity Field

Fig. 8 Flow fields for CFL number of 10,000 and Reynolds number equal to $10^{6}$. The SST (left) and DES (right) model outputs are qualitatively compared using pressure (top) and velocity (bottom) fields. 


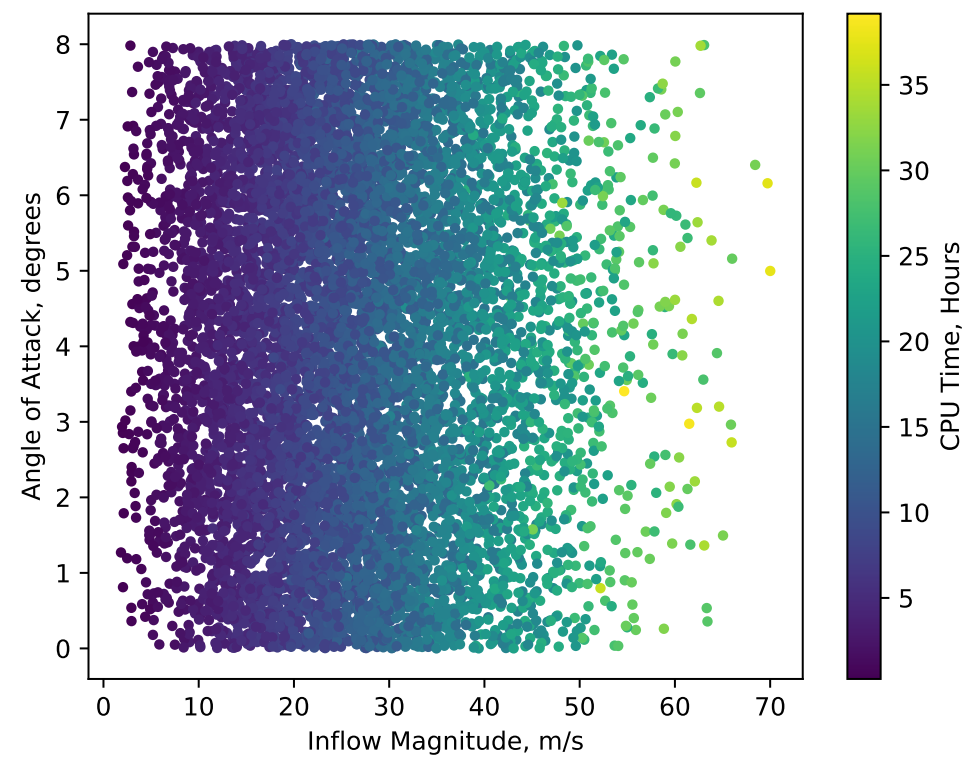

Fig. 9 Model evaluation time versus angle of attack versus inflow magnitude for the DES model using CFL=100.

\section{Results}

We examined the DU 91-W2-250 Airfoil in Nalu-Wind and compared the multilevel-multifidelity sampling approach to standard Monte Carlo. We chose this airfoil because it is representative of the midsection of a wind turbine blade. We decided to cast the turbulence model as the simulation fidelity and the CFL number as the discretization level parameter. We performed an initial set of trial samples and used this information to calculate the optimal allocation of model evaluations. In practice, we would then use this information to inform our full sampling efforts. In this study, since we had more than enough model evaluations across the different discretization levels and model fidelities, we compared the sample prescriptions calculated using the trial samples to that calculated using the previously mentioned full set of samples. We used this new full set of samples to construct a multilevel-multifidelity estimator, allocating the samples according to the $r_{l}$ and $N_{l} / N_{L}$ prescriptions for a computational budget of 60,000 CPU-hours. We also ran a separate set of samples of the truth model as a baseline reference. Each model displayed a wide range of computational cost, depending on the input parameters (see Figure 9 .

\section{A. Trial Results}

We first ran an initial set of trial sampling runs to inform our computational efforts. Parallel trial runs were launched for the SST and DES models, using CFL numbers of 100, 1,000, and 10,000, where each trial used the same random number generator seed. The trial function evaluations and associated $Y_{l}$ differences are plotted in Figures 10 and 11 . respectively. We applied Eqs. 8 and 9 to our data (see Table 1). Using this information, we calculated the optimal sample allocation for a computational budget of 60,000 hours (see Table 2).

Table 1 Summary of trial data set.

\begin{tabular}{ccccccccc}
\hline \hline CFL & SST Trials & DES Trials & $\mathbb{E}\left(C_{l}^{\mathrm{LF}}\right)(\mathrm{hr})$ & $\mathbb{E}\left(C_{l}^{\mathrm{HF}}\right)(\mathrm{hr})$ & $\rho_{l}^{2}$ & $\operatorname{Var}\left(Y_{l}^{\mathrm{HF}}\right)$ & $r_{l}$ & $N_{l}^{\mathrm{HF}} / N_{L}^{\mathrm{HF}}$ \\
\hline 10,000 & 195 & 189 & 0.28 & 0.29 & 0.9993 & $5,795,409.30$ & 36.75 & 994.36 \\
1,000 & 37 & 30 & 1.49 & 1.85 & 0.9998 & $19,389.46$ & 88.94 & 10.42 \\
100 & 5 & 5 & 8.90 & 9.23 & 0.9999 & $2,586.37$ & 138.82 & 1.00 \\
\hline \hline
\end{tabular}


Table 2 Prescribed sample allocations for a computational budget of 60,000 hours, based on the trial set of samples.

\begin{tabular}{ccc}
\hline \hline Level & $N_{l}^{\mathrm{LF}}(\tilde{C}=60,000 \mathrm{hr})$ & $N_{l}^{\mathrm{HF}}(\tilde{C}=60,000 \mathrm{hr})$ \\
\hline 10000 & 164393.0 & 4473.4 \\
1000 & 4167.8 & 46.9 \\
100 & 624.5 & 4.5 \\
\hline \hline
\end{tabular}

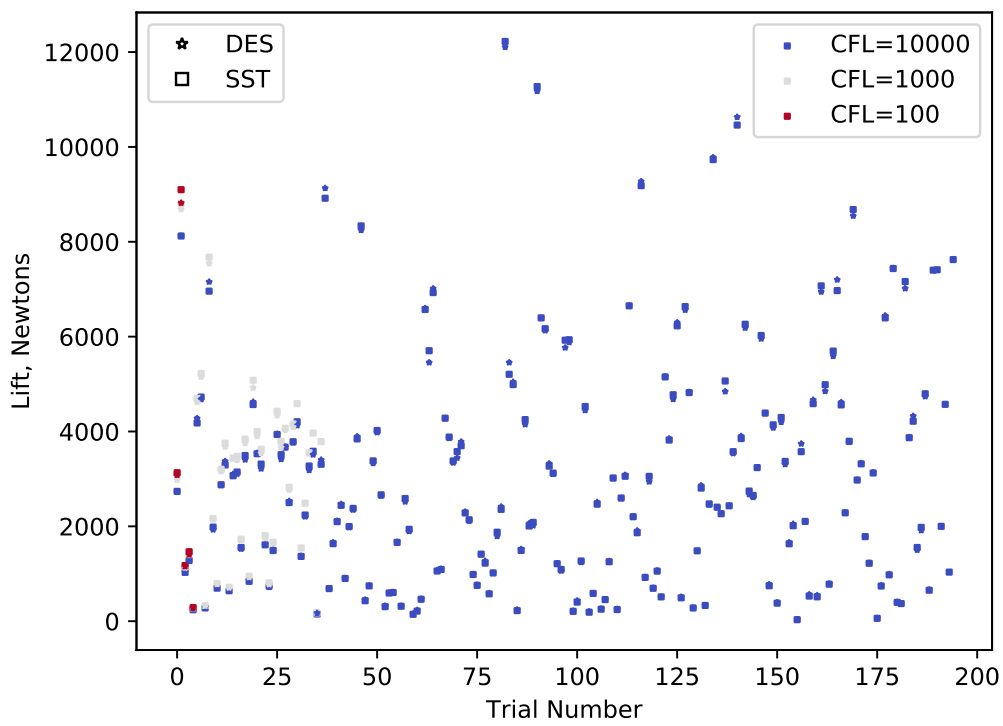

Fig. 10 Lift evaluation as a function of trial number, CFL number, and turbulence model.

\section{B. Completed Sampling Results}

We collected samples according to the prescriptions in Table 2 We used this collection of samples to re-calculate the optimal prescription of model evaluations, shown in Tables 3 and 4 . We use this sample allocation strategy moving forward. This allocation strategy is not radically different than the one calculated only using the trial samples.

Table 3 Summary of full data set.

\begin{tabular}{ccccccccc}
\hline \hline $\mathrm{CFL}$ & SST Trials & DES Trials & $\mathbb{E}\left(C_{l}^{\mathrm{LF}}\right)(\mathrm{hr})$ & $\mathbb{E}\left(C_{l}^{\mathrm{HF}}\right)(\mathrm{hr})$ & $\rho_{l}^{2}$ & $\operatorname{Var}\left(Y_{l}^{\mathrm{HF}}\right)$ & $r_{l}$ & $N_{l}^{\mathrm{HF}} / N_{L}^{\mathrm{HF}}$ \\
\hline 10,000 & 172,682 & 62,263 & 0.27 & 0.29 & 0.9999 & $5,732,626.29$ & 103.74 & 295.03 \\
1,000 & 12,053 & 374 & 1.47 & 2.04 & 0.9999 & $31,968.50$ & 124.40 & 7.83 \\
100 & 2,175 & 44 & 8.97 & 12.61 & 0.9999 & $2,912.33$ & 119.28 & 1.00 \\
\hline \hline
\end{tabular}

We swept the number of high-fidelity fine-discretization "truth" model evaluations from 1 to 5 , following the formula for optimal model evaluations and the ratios in Table 3 The resulting Monte Carlo convergence is plotted with respect to the number of "truth" model samples in Figure 12 The same data is plotted with respect to measured CPU-Time time in Figure 13 As expected, the multilevel-multifidelity estimator reduced the variance and computational burden of the problem. 


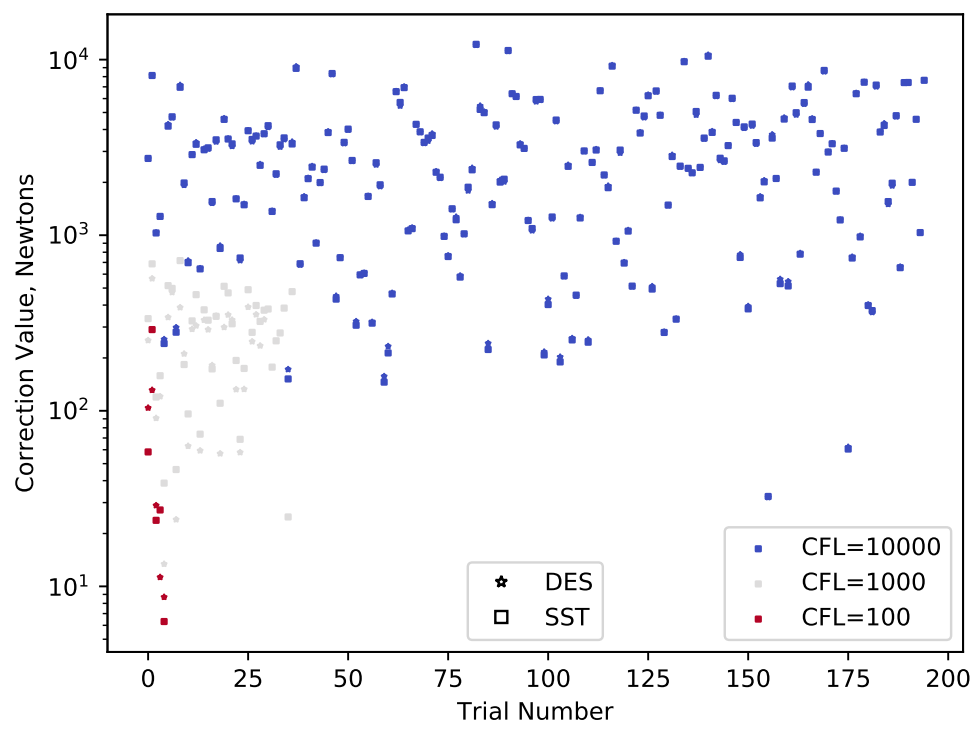

Fig. 11 Lift multilevel correction function plotted against trial number, CFL number, and turbulence model.

Table 4 Total number of samples used in the full analysis from $C F L=10,000$ and $C F L=1,000$. These include the trial runs, plus additional runs selected according to the model allocation ratios reported in Table 3 .

\begin{tabular}{ccc}
\hline \hline CFL & SST Trials & DES Trials \\
\hline 10,000 & $138,724.6$ & $1,337.2$ \\
1,000 & $4,417.9$ & 35.5 \\
100 & 540.6 & 4.5 \\
\hline \hline
\end{tabular}

\section{Comparrison of Estimated Errors}

We compare the estimated error and estimator standard deviation against the total computational burden for the multilevel-multifidelity estimator and compare it to standard Monte Carlo. We estimated the true expectation value using chaospy's stochastic collocation tool. We plot the estimated truth versus polynomial degrees in Figure 14 We decided to use fourth degree polynomials, as that is where this curve seems to saturate before it diverges.

The error plot is shown in Figure 15, which reflects the total computational cost due to increasing the number of truth runs from 1 to 5, along with the associated lower fidelity and other level model evaluations. The multilevel-multifidelity estimator is about one order of magnitude more accurate than the standard Monte Carlo approach. The estimators' standard deviation may be interpreted as a confidence interval. The error for each estimator is less than or is the same order of magnitude as the associated standard deviation, so we conclude that the estimators are not overconfident and that the error bounds interpretation is valid here. 


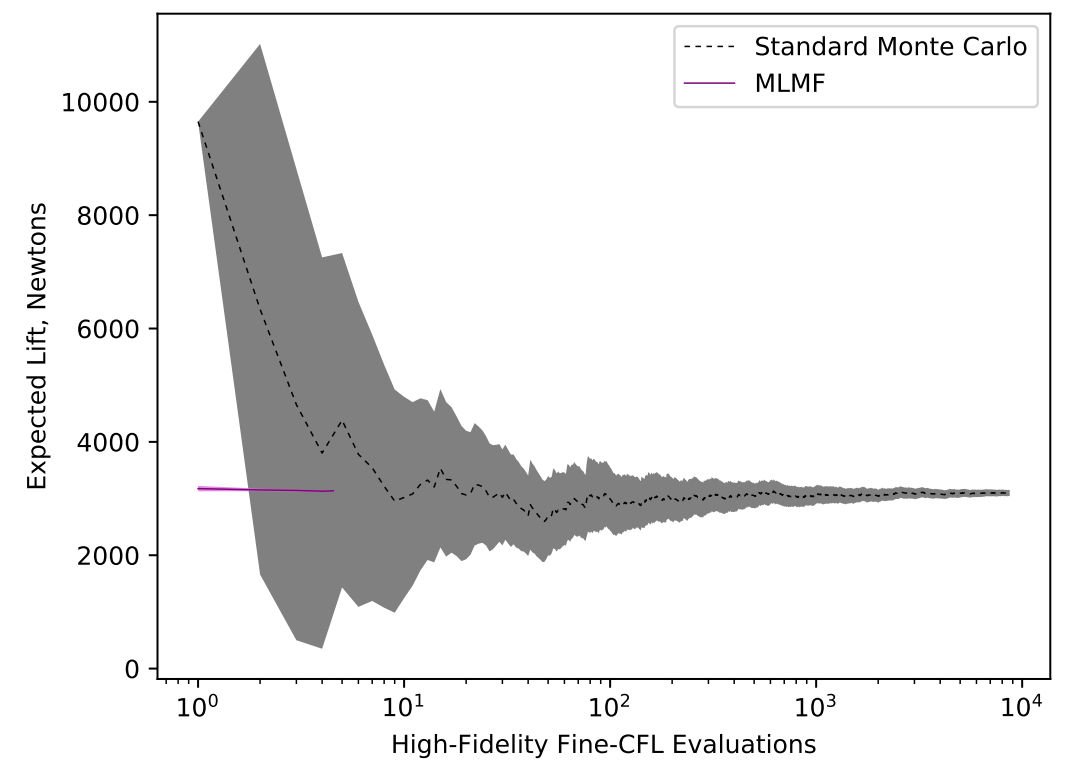

Fig. 12 Estimate of the average lift as a function of "truth" model evaluations. The clouds show two standard deviations using the estimator variance.

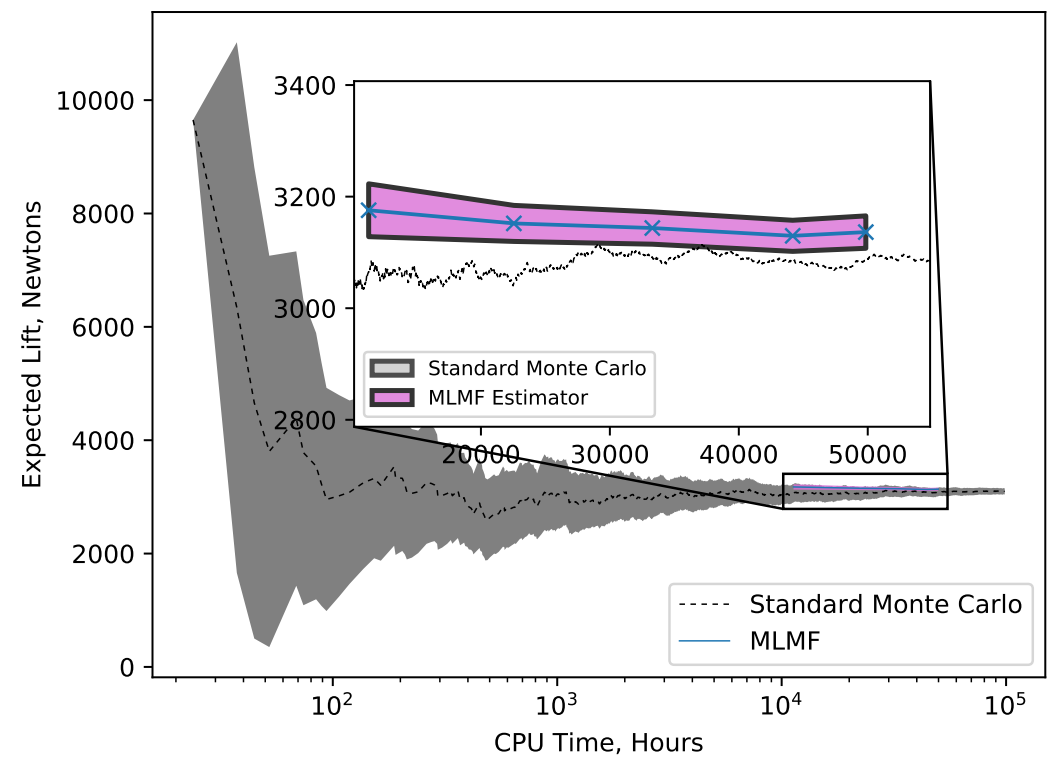

Fig. 13 Estimate of the average lift as a function of CPU time using the full set of samples. This reflects the total computational cost due to increasing the number of truth runs from 1 to 5 , along with the associated lower fidelity and other level model evaluations. The clouds show two standard deviations using the estimator variance. The dashed lines show two standard deviations of the final expectation estimated with the MLMF approach. 


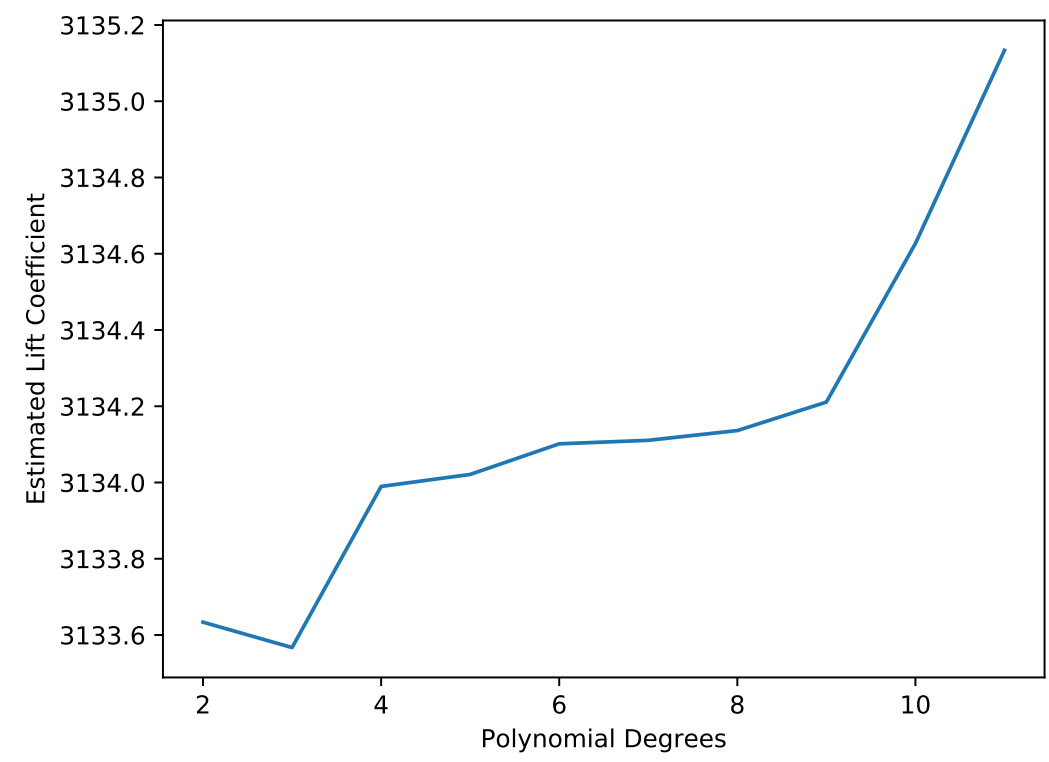

Fig. 14 Expected lift coefficient calcualted using stochastic collocation with Tikhonov regularization versus polynomial degrees

\section{Regular Monte Carlo $\quad \leftarrow$ MLMF}

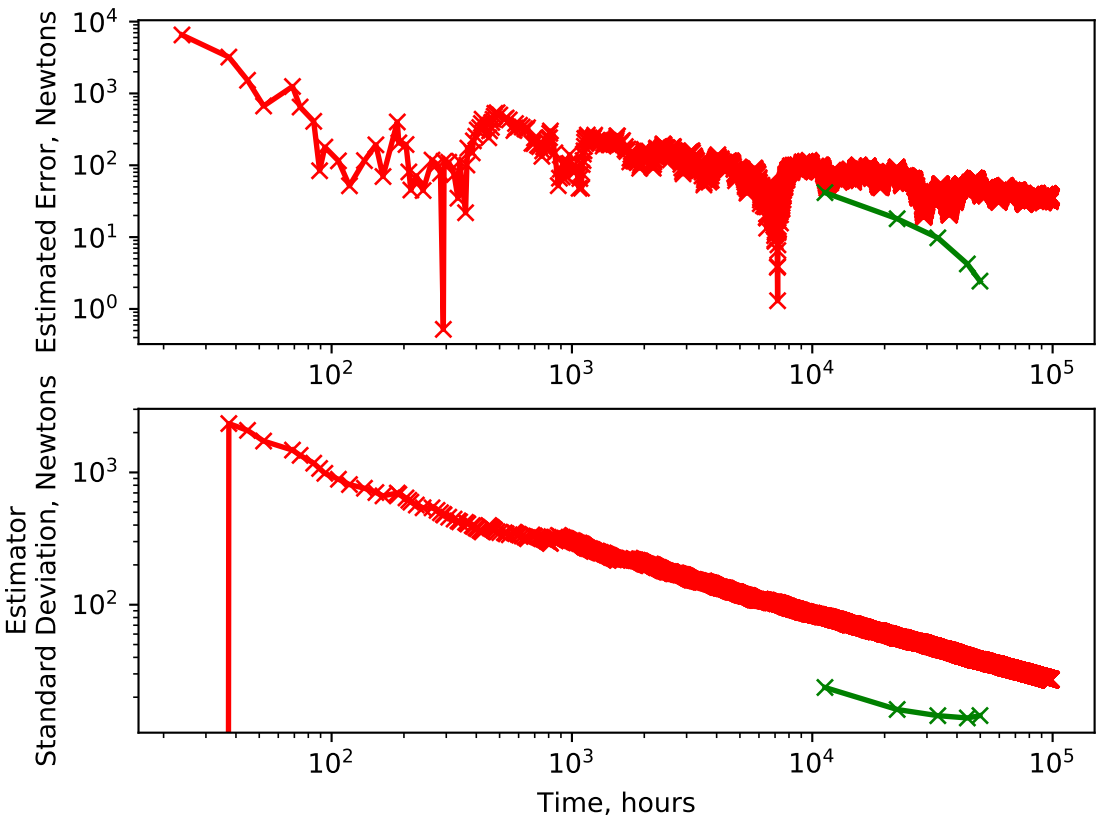

Fig. 15 Estimated error and estimator standard deviation plotted against time elapsed for the different Monte Carlo estimators considered. This reflects the total computational cost due to increasing the number of truth runs from min to max, along with the associated lower fidelity and other level model evaluations. 


\section{Discussion and Future Work}

It is well known that the error associated with Monte Carlo sampling is inversely proportional to the square root of the number of queried samples. It is natural to conclude from this that the error will scale with the inverse square root of the computational time because the computational demand for a simulation is proportional to the number of queried samples. To overcome this challenge, correlated models may be fused together to allow for very large numbers of Monte Carlo samples in relatively small computational time. In this study, we showed that the multifidelity approach can be used to fuse RANS and DES models of various temporal resolutions to reduce the computational burden of Monte Carlo simulation in a way that was formally independent of the number of dimensions. After our initial trial runs, we discovered that the optimal model allocation ratios strongly favored evaluating the low-fidelity model.

Our results are encouraging, indicating that standard Monte Carlo is not the most computationally efficient random sampling approach available when different model discretizations and fidelities are available. We spent on the order of $300 \mathrm{CPU}$ hours during the trial phase, where we estimated covariance and variance terms and constructed the difference functions. After the trial phase, we did not do any more high-fidelity fine-CFL model evaluations, spending the remainder of our computational budget evaluating lower-order models. We found that our multilevel-multifidelity estimators were not overconfident or biased. Using the full multilevel-multifidelity approach, we obtained an order of magnitude reduction in computation cost to achieve an equivalent estimator standard deviation as a traditional Monte Carlo approach.

The simulations displayed a wide range of computational time requirements. We launched several parallel jobs to each perform several samples, intending to collect all the samples after the jobs finished. However, this creates a natural filter; parameters associated with smaller evaluation times will be sampled more using this method. So, if we collect all samples from each queue, less computationally demanding models (i.e., lower-resolution ones) would not be sampled identically to the more computationally-demanding models because this time-filtering effect would be more extreme for more computationally demanding models. To counter this issue, we only used the first $N$ samples from every parallelized sampling program, where $N$ is the minimum number of completed samples across all parallelized programs.

It is interesting to note that the "multifidelity" control variate aspect of this approach relies on a large difference between the number of low- and high-fidelity model evaluations, whereas the "multilevel" aspect of the approach has no such assumption. The difference in computational cost between the SST and DES models was of order one while the difference in computational demand between the different CFL numbers was about one order of magnitude. In practice, it would have been more practical to discard the SST model in this case, using a purely multilevel approach, or selecting a different model like XFOIL, Euler equations, or a coarser grid as the low-fidelity model. In future work, we plan to apply this technique to efficiently propagate realistic uncertainties in operational conditions when modeling wind power plants.

\section{Acknowledgments}

The research was performed using computational resources sponsored by the U.S. Department of Energy Office of Energy Efficiency and Renewable Energy and located at the National Renewable Energy Laboratory. This work was authored [in part] by the National Renewable Energy Laboratory, operated by Alliance for Sustainable Energy, LLC, for the U.S. Department of Energy (DOE) under Contract No. DE-AC36-08GO28308. Funding provided by the U.S. Department of Energy Office of Energy Efficiency and Renewable Energy Wind Energy Technologies Office. The views expressed in the article do not necessarily represent the views of the DOE or the U.S. Government. The U.S. Government retains and the publisher, by accepting the article for publication, acknowledges that the U.S. Government retains a nonexclusive, paid-up, irrevocable, worldwide license to publish or reproduce the published form of this work, or allow others to do so, for U.S. Government purposes.

\section{References}

[1] Dunn, M. C., Shotorban, B., and Frendi, A., "Uncertainty quantification of turbulence model coefficients via Latin hypercube sampling method," Journal of Fluids Engineering, Vol. 133, No. 4, 2011, p. 041402.

[2] Witteveen, J., Doostan, A., Pecnik, R., and Iaccarino, G., "Uncertainty quantification of the transonic flow around the RAE 2822 airfoil," Center for Turbulence Research, Annual Briefs, Stanford University, 2009.

[3] Padron, A. S., Alonso, J. J., Palacios, F., Barone, M. F., and Eldred, M. S., "Multi-fidelity uncertainty quantification: application 
to a vertical axis wind turbine under an extreme gust," 15th AIAA/ISSMO Multidisciplinary Analysis and Optimization Conference, 2014, p. 3013.

[4] Ng, L. W.-T., and Eldred, M., "Multifidelity uncertainty quantification using non-intrusive polynomial chaos and stochastic collocation," 53rd AIAA/ASME/ASCE/AHS/ASC Structures, Structural Dynamics and Materials Conference 20th AIAA/ASME/AHS Adaptive Structures Conference 14th AIAA, 2012, p. 1852.

[5] Peherstorfer, B., Willcox, K., and Gunzburger, M., "Survey of multifidelity methods in uncertainty propagation, inference, and optimization," Tech. rep., Aerospace Computational Design Laboratory, Department of Aeronautics \& Astronautics, Massachusetts Institute of Technology, 2016.

[6] Eldred, M. S., Ng, L. W., Barone, M. F., and Domino, S. P., "Multifidelity uncertainty quantification using spectral stochastic discrepancy models," Handbook of Uncertainty Quantification, Springer, 2015, pp. 1-45.

[7] Wang, J.-X., Roy, C. J., and Xiao, H., "Propagation of input uncertainty in presence of model-form uncertainty: a multifidelity approach for computational fluid dynamics applications," ASCE-ASME Journal of Risk and Uncertainty in Engineering Systems, Part B: Mechanical Engineering, Vol. 4, No. 1, 2018, p. 011002.

[8] Pisaroni, M., Nobile, F., and Leyland, P., "Continuation multi-level Monte-Carlo method for uncertainty quantification in turbulent compressible aerodynamics problems modeled by RANS,” Tech. rep., MATHICSE Technical Report 10.2017, 2017.

[9] Peherstorfer, B., Willcox, K., and Gunzburger, M., "Optimal model management for multifidelity Monte Carlo estimation," SIAM Journal on Scientific Computing, Vol. 38, No. 5, 2016, pp. A3163-A3194.

[10] Fairbanks, H. R., Doostan, A., Ketelsen, C., and Iaccarino, G., "A low-rank control variate for multilevel Monte Carlo simulation of high-dimensional uncertain systems," Journal of Computational Physics, Vol. 341, 2017, pp. 121-139.

[11] Geraci, G., Eldred, M., and Iaccarino, G., "A multifidelity control variate approach for the multilevel Monte Carlo technique," Center for Turbulence Research Annual Research Briefs, 2015.

[12] Geraci, G., Eldred, M. S., and Iaccarino, G., "A multifidelity multilevel Monte Carlo method for uncertainty propagation in aerospace applications," 19th AIAA Non-Deterministic Approaches Conference, 2017, p. 1951.

[13] Nalu-Wind Documentation, https://nalu-wind.readthedocs.io/en/latest/index.html retrieved on 12/7/2018, version: https://github.com/Exawind/nalu-wind/commit/72e4a647ba8626a654b435e738ae0ebcfff6b31e

[14] Domino, S., "Sierra low mach module: Nalu theory manual 1.0," SAND2015-3107W, Sandia National Laboratories Unclassified Unlimited Release (UUR), 2015. https://github.com/NaluCFD/NaluDoc.

[15] Monschke, J. A., and Eldred, M. S., "Multilevel-multifidelity acceleration of PDE-constrained optimization," 58th AIAA/ASCE/AHS/ASC Structures, Structural Dynamics, and Materials Conference, 2017, p. 0132.

[16] Ng, L. W., and Willcox, K. E., "Multifidelity approaches for optimization under uncertainty," International Journal for numerical methods in Engineering, Vol. 100, No. 10, 2014, pp. 746-772.

[17] Scholcz, T. P., "Multi-fidelity methods for fluid-structure interaction and uncertainty quantification," Ph.D. thesis, Delft University of Technology, 2015. URL http://resolver.tudelft.nl/uuid:808f8eb8-43e7-4c69-b20d-f68e75c07b7a

[18] Fusi, F., Guardone, A., Quaranta, G., and Congedo, P. M., "Multifidelity physics-based method for robust optimization applied to a hovering rotor airfoil," AIAA Journal, Vol. 53, No. 11, 2015, pp. 3448-3465.

[19] Maniaci, D. C., Frankel, A. L., Geraci, G., Blaylock, M. L., and Eldred, M. S., "Multilevel uncertainty quantification of a wind turbine large eddy simulation model," 7th European Conference on Computational Fluid Dynamics, 2018.

[20] Geraci, G., Eldred, M. S., and Iaccarino, G., "A multifidelity multilevel Monte Carlo method for uncertainty propagation in aerospace applications,” 19th AIAA Non-Deterministic Approaches Conference, 2017, p. 1951.

[21] Geraci, G., Eldred, M., and Iaccarino, G., "A multifidelity control variate approach for the multilevel Monte Carlo technique," Center for Turbulence Research Annual Research Briefs, 2015.

[22] Feinberg, J., and Langtangen, H. P., "Chaospy: An open source tool for designing methods of uncertainty quantification," Journal of Computational Science, Vol. 11, 2015, pp. 46 - 57. doi:https://doi.org/10.1016/j.jocs.2015.08.008, URL http: //www.sciencedirect.com/science/article/pii/S1877750315300119 
[23] Haering, S. W., "Anisotropic hybrid turbulence modeling with specific application to the simulation of pulse-actuated dynamic stall control,", 2015. doi:10.15781/T2TM3V.

[24] Adams, B. M., Ebeida, M. S., Eldred, M. S., Geraci, G., Jakeman, J. D., Maupin, K. A., Monschke, J. A., Stephens, J. A., Swiler, L. P., Vigil, D. M., Wildey, T. M., Bohnhoff, W. J., Dalbey, K. R., Eddy, J. P., Frye, J. R., Hooper, R. W., Hu, K. T., Hough, P. D., Khalil, M., Ridgway, E. M., Winokur, J. G., and Rushdi, A., "DAKOTA, a multilevel parallel object-oriented framework for design optimization, parameter estimation, uncertainty quantification, and sensitivity analysis,” Tech. rep., 2018. 